Published in final edited form as:

ACS Chem Biol. 2016 December 16; 11(12): 3319-3327. doi:10.1021/acschembio.6b00759.

\title{
Domain Organization and Active Site Architecture of a Polyketide Synthase $C$-methyltransferase
}

\author{
Meredith A. Skiba ${ }^{1,2}$, Andrew P. Sikkema ${ }^{1,2}$, William D. Fiers ${ }^{3}$, William H. Gerwick ${ }^{4,5}$, David \\ H. Sherman ${ }^{1,6,7,8}$, Courtney C. Aldrich ${ }^{3}$, and Janet L. Smith ${ }^{1,2,{ }^{*}}$ \\ ${ }^{1}$ Life Sciences Institute, University of Michigan, Ann Arbor, MI \\ ${ }^{2}$ Department of Biological Chemistry, University of Michigan, Ann Arbor, MI \\ ${ }^{3}$ Department of Medicinal Chemistry, University of Minnesota, Minneapolis, MN \\ ${ }^{4}$ Center for Marine Biotechnology and Biomedicine, Scripps Institution of Oceanography, \\ University of California San Diego, La Jolla, CA \\ ${ }^{5}$ School of Pharmacy and Pharmaceutical Sciences, University of California San Diego, La Jolla, \\ CA \\ ${ }^{6}$ Department of Medicinal Chemistry, University of Michigan, Ann Arbor, MI \\ ${ }^{7}$ Department of Chemistry, University of Michigan, Ann Arbor, MI \\ ${ }^{8}$ Department of Microbiology and Immunology, University of Michigan, Ann Arbor, MI
}

\begin{abstract}
Polyketide metabolites produced by modular type I polyketide synthases (PKS) acquire their chemical diversity through the variety of catalytic domains within modules of the pathway. Methyltransferases are among the least characterized of the catalytic domains common to PKS systems. We determined the domain boundaries and characterized the activity of a PSS $C$ methyltransferase ( $C$-MT) from the curacin A biosynthetic pathway. The $C$-MT catalyzes $S$ adenosylmethionine-dependent methyl transfer to the a-position of $\beta$-ketoacyl substrates linked to acyl carrier protein (ACP) or a small-molecule analog, but does not act on $\beta$-hydroxyacyl substrates or malonyl-ACP. Key catalytic residues conserved in both bacterial and fungal PKS $C$ MTs were identified in a 2- $\AA$ crystal structure and validated biochemically. Analysis of the structure and the sequences bordering the $C$-MT provides insight into the positioning of this domain within complete PKS modules.
\end{abstract}

\section{Graphical abstract}

\footnotetext{
*To whom correspondence should be addressed at Life Sciences Institute, University of Michigan, 210 Washtenaw Ave., Ann Arbor, MI 48109; JanetSmith@umich.edu.

Supporting Information

Supporting Information methods, tables, and figures. This material is available free of charge via the internet at http://pubs.acs.org.

Accession Codes

Atomic coordinates and structure factors have been deposited in the Protein Data Bank: 5THY (SeMet CurJ $C$-MT), 5 THZ (wild type CurJ $C$-MT).
} 

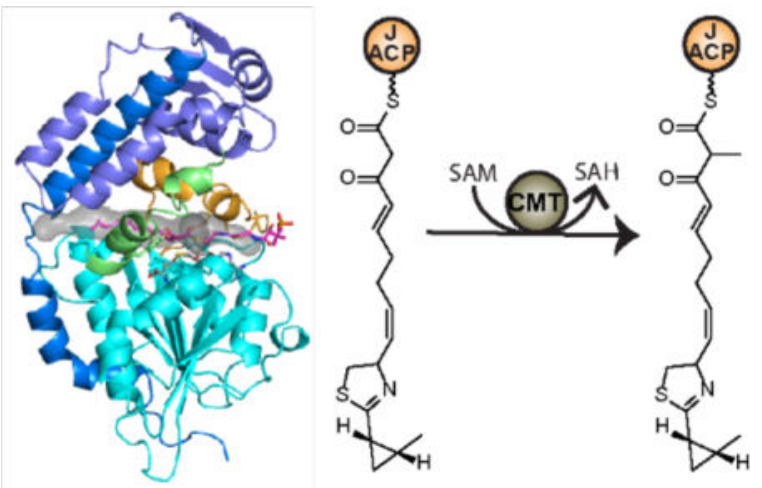

\section{Introduction}

Polyketides and their derivatives are among the most chemically and biologically diverse natural products, and their biosynthetic pathways contain a wealth of synthetic potential.

Type I polyketide synthases (PKSs) are large multifunctional enzymes that generate complex polyketides with exquisite stereo and regioselectivity ${ }^{1-3}$. Typical type I PKSs contain acyltransferase (AT) and ketosynthase (KS) domains for the selection of acyl-CoA building blocks and extension of polyketide intermediates as well as an acyl carrier protein (ACP) domain to shuttle intermediates between domains. Additionally, PKSs may have one or more modification domains, such as a ketoreductase (KR), dehydratase (DH), enoylreductase (ER) and methyltransferase (MT), for further processing of the $\beta$-ketoacyl-ACP product of KS extension (Figure 1a). In bacteria, type I PKSs are commonly found as modular assembly lines, where each module catalyzes a single two-carbon extension and modification of the polyketide and then passes the intermediate to the next module for further elongation. In some cases, PKS modules lack embedded AT domains and rely on in trans ATs encoded in the biosynthetic gene cluster for the delivery of acyl-CoAs. Fungal type I PKSs typically occur as single modules that catalyze multiple rounds of chain elongation and modification ${ }^{1,4}$.

Methyl groups can be introduced at the a-position of a polyketide intermediate through the incorporation of methylmalonyl-CoA extender units selected by the AT or through the action of embedded $C$-methyltransferase ( $C$-MT) domains. $S$-adenosylmethionine (SAM)dependent PKS $C$-MT domains were initially characterized in the lovastatin biosynthetic pathway of the fungus Aspergillus terreus and the mixed PKS / non-ribosomal peptide synthetase (NRPS) yersiniabactin pathway ${ }^{5-7}$ and subsequently annotated in multiple fungal PKSs as well as in bacterial cis-AT and trans-AT pathways ${ }^{8-16}$.

PKS $C$-MTs are members of the class I MT superfamily ${ }^{17,}{ }^{18}$, identified by six sequence motifs in the SAM-binding core ${ }^{19}$. Despite structural conservation of the MT core, class I MTs lack conserved active site residues due to the intrinsic reactivity of SAM and the great variety of methyl acceptor substrates. Moreover, numerous loop and domain insertions to the MT core facilitate substrate binding or oligomer formation, resulting in an extremely divergent superfamily with very low overall sequence identity (generally below $20 \%$ ). $C$ MTs are the only domains commonly found within PKS modules that have remained 
structurally uncharacterized. Beyond the easily recognized sequence motifs for SAM binding, the PKS $C$-MT domain boundaries are uncertain. However, a pseudomethyltransferase ( $\Psi$-MT) domain is present in the PKS homolog, the metazoan fatty acid synthase (mFAS) ${ }^{20}$, but the $\Psi$-MT lacks the SAM binding motifs and appears to be significantly shorter than the PKS $C$-MTs.

$C$-MTs could potentially act on the unmodified a-carbon at any point during the PKS catalytic cycle, unlike KS, KR, DH and ER domains, which act in a specific sequence (Figure 1b). In an earlier study, it was proposed that PKS $C$-MT domains act after KS condensation and prior to subsequent reductive modifications based on the accumulation of a 2-methyl-1,3-diketo-containing shunt product in a mutagenized lovastatin producing fungus (Figure $1 \mathrm{~b}$, Route 1$)^{6}$. Methylation of the $\beta$-ketoacyl intermediate via Route 1 was observed directly in two fungal PKSs ${ }^{14,21}$. However, two bacterial PKS $C$-MTs that dimethylate the a-position of polyketide intermediates were shown to act exclusively via Route 2 in epothilone biosynthesis (Figure 1b, Route 2) and either on the $\beta$-ketoacyl intermediate of Route 1 or on malonyl-ACP preceding KS condensation in yersiniabactin biosynthesis $^{22,23}$. Thus it was unclear when monomethylation occurs in modular $C$-MTs. Recent work demonstrated $C$-MT activity via Route 1 in bacterial trans-AT and cis-AT pathways 24,25 .

The biosynthetic pathway for the antimitotic polyketide curacin A (Figure 1a) produced by the marine cyanobacterium Moorea producens has provided a wealth of structural insight into conventional and unusual PKS biosynthetic capabilities ${ }^{8,26-35}$. Modules CurG-CurM catalyze typical PKS extension reactions. Like many PKS pathways from Moorea species, a $C$-MT domain in CurJ installs a single a-methyl (Figure 1a). Here we report the crystal structure of CurJ $C$-MT at $2.1 \AA$ resolution and demonstrate that CurJ $C$-MT follows Route 1 by methylating the a-position of the $\beta$-keto acyl substrate. The structure reveals key conserved features of PKS $C$-MT active sites and provides important clues about how $C$ MTs are positioned within PKS modules.

\section{Results and Discussion}

\section{Identification of C-MT domain boundaries}

$C$-MT domains within PKS modules have been annotated through identification of MT motifs (Supplementary Figure 1). However, all have a large unannotated region between the preceding catalytic domain and MT motif I, for example 228 amino acids following the DH domain of CurJ. Moreover $C$-MTs from cis-AT and trans-AT PKS pathways cluster separately in a phylogenetic tree (Supplementary Figure 2) ${ }^{15}$, and have distinct positions within PKS modules, typically preceding KR domains in cis-AT pathways and following KR domains in trans-AT pathways.

The $C$-MT N-terminal extension is less conserved than the MT core (for example, $30 \%$ identity for the CurJ N-terminal extension and $50 \%$ for the MT core compared to the homologous regions of the GphI $C$-MT from the myxobacterial gephyronic acid pathway ${ }^{15}$ ). However, the N-terminal extension is likely to have a defined structure, as it is highly conserved among cyanobacterial $C$-MTs, (for example, $78 \%$ identity to CrpA from Nostoc 
sp. ATCC $53780^{10}$ ). We identified approximate domain boundaries based on a multiple sequence alignment of $C$-MTs from cyanobacterial PKS pathways, created fifteen constructs encoding the CurJ $C$-MT with various $\mathrm{N}$ - and C-termini (Table S4), and tested twelve for production of soluble protein. Ultimately, CurJ residues 1269-1658, herein referred to as 1390, yielded a pure, stable, recombinant $C$-MT domain that was monomeric in solution as determined by size exclusion chromatography (data not shown).

\section{Methyltransferase Activity}

Based on precedent for $C$-methylation in PKSs, we expected that CurJ $C$-MT would act on either malonyl-ACP via Route 2 or the $\beta$-ketoacyl intermediate formed after KScondensation via Route 1 (Figure 1b) ${ }^{14,21,23-25}$. As native CurJ substrates were unavailable, acetoacetyl-ACP (1) was tested using an assay based on intact protein mass spectrometry (MS). Complete conversion of acetoacetyl-ACP (1) to a-methyl-acetoacetyl-ACP (2) (Figure 2a) was observed by LC-MS. However, no methylation occurred with malonyl-ACP (3) (Figure 2c), establishing that CurJ follows Route 1 and not Route 2.

We also tested a potential methylation Route 3 where the $C$-MT acts after KR reduction of the $\beta$-ketoacyl intermediate, i.e. on the $\beta$-hydroxyacyl intermediate. Reactions performed with DL- $\beta$-hydroxybutyryl-ACP (4) to mimic the $\beta$-hydroxy intermediate failed to generate a methylated product (Figure 2e), indicating that the $C$-MT is specific for the $\beta$-ketoacyl intermediate. CurJ $C$-MT also methylated an $N$-acetyl cysteamine (NAC-) linked $\beta$ ketopentanoyl substrate (5) (Figure 2 b) to (6), but not $\beta$-hydroxypentanoyl-NAC (7) (Figure $2 d)$.

Methylation of the $\beta$-ketoacyl intermediate via Route 1 is the most thermodynamically favorable for the formation of the carbanion nucleophile. Among the potential substrates via Routes 1, 2 and 3, the $\beta$-ketoacyl intermediate has the lowest $\mathrm{pKa} \sim 10$ due to the flanking carbonyls. By contrast, the pKa of the a-proton in malonyl-ACP is several units higher as a result of the carboxylate anion. Additionally, the $\beta$-hydroxy intermediate produced by the $\mathrm{KR}$ has the least acidic a-proton with a pKa estimated $\sim 21$, making it the least favorable substrate for methyl transfer.

\section{Structure of CurJ C-MT}

The crystal structure of selenomethionine-labeled CurJ $C$-MT was solved to $2.1 \AA$ by singlewavelength anomalous diffraction (SAD) phasing (Figure 3 and Table 1). The CurJ $C$-MT has a class I MT core domain for SAM binding (residues 1-27, 172-306, 342-381), comprised of a seven-stranded $\beta$-sheet flanked by $a$-helices. A 32-residue insertion (307341 , including helix 13) between $\beta$-strand 8 and helix 14 of the MT core distinguishes the PKS $C$-MT from a minimal class I MT (Figure 3). The "core insertion" extends over the active site, and is conserved in PKS $C$-MTs from cis-AT (49\% identity to GphI $C$-MT), trans-AT (44\% to RhiB) and fungal PKS pathways (44\% to SQTKS) (Supplementary Figure 1). The mFAS $\Psi$-MT has a homologous 26 -residue insertion in the equivalent position (Supplementary Figure 4). 
Much of the unannotated N-terminal extension of CurJ $C$-MT forms a helical "lid" over the MT core. The large lid (residues 28-171) includes eight helices and a three-stranded $\beta$-sheet and is connected to the core by a "lid-to-core junction" containing helices 7 and 8 (Figure 3). Helices 1 and 2 (Figure 3) form a long amphipathic N-terminal helical "seatbelt" (residues 9-54) that wraps around the MT core and extends into the lid. Nearly all other $C$-MTs from bacterial cis-AT pathways contain a lid of similar length with an amphipathic sequence at the N-terminus (Supplementary Figure 1), suggesting that the seatbelt is present in nearly all of them. The seatbelt serves an architectural function of bringing together the $\mathrm{N}$ - and Ctermini at the bottom of the core domain ( $15 \AA$ separation). This is analogous to the mFAS $\Psi$-MT, which lacks helix 1 of the seatbelt and instead contributes a $\beta$-strand to the core $\beta$ sheet, bringing the domain termini within $\sim 20 \AA$ of one another (Supplementary Figure 4). The mFAS $\Psi$-MT lid is a truncated variant of the PKS $C$-MT lid.

Knowledge of the $C$-MT domain boundaries within CurJ allowed us to locate a key structure element of the CurJ KR domain (Figure 4). An extended $\beta$-ribbon is an integral part of PKS $\mathrm{KR}$ domains, which are comprised of structural $\left(\mathrm{KR}_{\mathrm{S}}\right)$ and catalytic $\left(\mathrm{KR}_{\mathrm{C}}\right)$ sub-domains ${ }^{36}$. The extended $\beta$-ribbon contributes to the $\beta$-sheets of both $\mathrm{KR}_{\mathrm{S}}$ and $\mathrm{KR}_{\mathrm{C}}$, but its two $\beta$ strands lie within inter-domain linker sequences, generally flanking the $\mathrm{KR}_{\mathrm{S}}$ subdomain $^{36-45}$. However, in CurJ the highly conserved first $\beta$-strand of the KR $\beta$-ribbon does not flank $\mathrm{KR}_{\mathrm{S}}$, but is located between the DH and $C$-MT domains (amino acids 1254-1261, nine residues following the $\mathrm{DH}$ and eight residues preceding the $C$-MT, Figure 4 ). Interestingly, the first $\beta$-strand of the mFAS KR $\beta$-ribbon is located in the analogous location between DH and $\Psi$-MT domains. Together with the similarity of the $C$-MT and $\Psi$ MT structures and the similar positions of their $\mathrm{N}$ - and $\mathrm{C}$-termini, this remarkable evolutionary conservation is a strong indication that the modifying region of $\mathrm{CurJ}$ ( $\mathrm{DH}-C$ MT-KR-ACP) and other PKS modules is organized similarly to the modifying region of mFAS (DH- $\left.\Psi-\mathrm{MT}-\mathrm{KR}_{\mathrm{S}}-\mathrm{ER}-\mathrm{KR}_{\mathrm{C}}-\mathrm{ACP}\right)$, and that the structure of $\mathrm{mFAS}^{20}$ can guide modeling of the PKS module modifying region (Figure $4 \mathrm{a}$ ).

\section{Active Site Architecture}

A hydrophobic tunnel between the MT core and the lid is long enough to accommodate the full CurJ $C$-MT substrate (Figure 3b), consistent with the observed activity on the hydrophobic $\beta$-ketoacyl substrate via Route 1 , and the lack of activity with the negatively charged malonyl-ACP. $S$-adenosyl-L-homocysteine (SAH) was bound in the MT core below the tunnel, supported by clear electron density (Supplementary Figure 5). The conserved side chains of His 280 and Glu306 form a hydrogen-bonded dyad $8 \AA$ from the SAH (Figure 5). The His-Glu dyad is invariant in all bacterial and fungal PKS $C$-MTs, thus the imidazole may act as a catalytic base to deprotonate the substrate a-carbon and form a carbanion nucleophile to facilitate the MT reaction. The reactivity of the His-Glu dyad may be enhanced by the surrounding hydrophobic environment (conserved Ala281, Phe318, Trp324, Pro337, Leu338, Trp344). Additionally, conserved Tyr169 is $\sim 4 \AA$ from the presumed SAM sulfonium position, and may facilitate methyl transfer through a $\mathrm{CH}-\mathrm{O}$ hydrogen bond with the SAM methyl ${ }^{46}$. The invariant Asn277 side chain points into the active site between SAH and the His-Glu dyad. Given its conservation and proximity to the SAM sulfonium, Asn277 could play a role in substrate positioning. 
Remarkably, the SAH homocysteine and nearby amino acids are the least well ordered regions of the active site. The two slightly different crystal forms (Table 1) provided four independent views of the $C$-MT and revealed small differences in position for the $\mathrm{SAH}$ homocysteine, helix 8 in the lid-to-core junction, and MT motif I, which coordinates the SAM/SAH carboxylate (Figure 6, Supplementary Figure 5). For two of the four $C$-MT views, density indicated partial disorder for Tyr169 in helix 8 and for the SAH homocysteine, and multiple positions for Thr208 in motif I. In contrast, the SAH adenosine and His-Glu dyad are identically positioned and supported by strong density in all four views. The His 280 position is stabilized by two hydrogen bonds within the 277-282 loop and by backbone hydrogen bonds with the conserved Arg333 side chain in the core insertion (Figure 5). These observations indicate that the substrate-binding region of the active site is flexible in absence of $\beta$-ketoacyl-ACP.

The CurJ $C$-MT core loops, the core insertion, and the lid-to-core junction surround the entrance to the active site, and amino acids in these regions are likely to interact with the ACP (Figure 3b, Figure 5). An analogous core insertion exists in the only other structurally characterized SAM-dependent enzyme with an ACP-linked substrate, cyclopropane synthase of mycolic acid biosynthesis ${ }^{47}$. As with cyclopropane synthase, the position and the highly conserved sequence of the PKS $C$-MT core insertion are consistent with a role in ACP recognition.

\section{Activity of CurJ $C$-MT with active site substitutions}

To probe the role of conserved amino acids in the active site, CurJ $C$-MT variants were produced and activity was evaluated using a phosphopantetheine ejection mass spectrometry assay (Figure 2f, Supplementary Figure 6$)^{48}$. Substitutions in the active site His-Glu dyad abolished activity, consistent with their proposed role as a catalytic dyad. The melting temperatures of the wild type and the His variants were comparable (Supplementary Table 2 ), indicating that the reduced activity was not due to a decrease in the structural integrity of the protein. A Y169F variant had three-fold reduced activity, in agreement with a role in facilitating the methyltransfer reaction. The N277A variant reduced activity two fold suggesting that it plays a role in substrate recognition or positioning in the active site.

\section{Substrate Modeling}

His208, the strongest candidate for a catalytic base, is $8 \AA$ from the SAM sulfonium on the opposite side of the active site. We modeled the full-length CurJ $C$-MT substrate into the active site tunnel, as attempts to soak small molecule NAC substrates $(\mathbf{5}, \mathbf{7})$ yielded additional but uninterpretable density at the presumed substrate binding site between SAH and His280. The full length CurJ $C$-MT substrate was docked between the MT core and lid domain and manually edited to orient the a-carbon in line with the sulfonium (Figure 3b, Figure 5). While the tunnel can accommodate the full substrate, the sulfonium and His 280 were too far apart for both to interact with the a-carbon of the $\beta$-ketoacyl intermediate (Figure 5). Additionally, hydrogen-bonding partners that could stabilize an enolate intermediate were not apparent. Thus, we propose that $\beta$-ketoacyl-ACP binding leads to small movements of the flexible active site elements (lid-to-core junction, core insertion and adjacent loops) and positions the substrate a-carbon, SAM methionine, Tyr169 and enolate- 
stabilizing hydrogen bonding partners closer to the His-Glu dyad and in optimal positions for methyl transfer. Due to the open active site in the crystal structures, we are unable to predict the stereochemical outcome of the reaction. Additionally, the acidity of the newly formed stereogenic proton precludes chemoenzymatic assignment. The chiral center in the CurJ $C$-MT product is eliminated by action of the CurJ DH, which catalyzes formation of a vinyl-methyl functionality in the curacin polyketide chain.

\section{Similarities of CurJ $\boldsymbol{C}$-MT to other PKS $\boldsymbol{C}$-MTs}

The CurJ $C$-MT structure is an excellent model for a pre-catalytic state of $C$-MTs embedded in PKS modules. These PKS MTs share several distinguishing features including the active site His-Glu dyad, the lid-to-core junction helices, and the MT core insertion (Supplementary Figure 1). Overall, $C$-MTs from trans-AT pathways and fungal iterative PKSs have lid domains approximately 30 residues shorter than the CurJ $C$-MT and others from cis-AT pathways (Figure 3, Supplementary Figure 1). Some PKS $C$-MTs produce gemdimethyl products ${ }^{12,23,25}$, which the spacious $C$-MT active site could facilitate by substrate re-orientation between methyl-transfer reactions. Although the natural CurJ $C$-MT substrate is quite long, the domain was capable of methylating shorter $\beta$-keto acyl substrates, such as acetoacetyl-ACP. This is in contrast to the fungal LovB $C$-MT, which was selective for substrates of similar length to the native substrate and had no activity on an acetoacetyl analog 21 . Increased substrate specificity in iterative fungal $C$-MTs relative to bacterial type I PKS $C$-MTs can be rationalized, as fungal $C$-MTs have evolved to be active in specific rounds of the iterative catalytic cycle, whereas PKS $C$-MTs will encounter only one $\beta$ ketoacyl-ACP during the single round of catalysis within the module.

The biochemical and structural characterization of the CurJ $C$-MT is consistent with previous findings that monomethylating PKS $C$-MTs act on $\beta$-ketoacyl intermediates following KS mediated chain elongation, and further shows no activity with $\beta$-hydroxyacyl intermediates. Additionally, the structure provides new insights regarding the mechanism of a-methylation in both bacterial and fungal PKS $C$-MTs, including identification of a conserved His-Glu dyad and other active site elements that are flexible in absence of the methyl acceptor substrate. Similarities of the CurJ $C$-MT and mFAS $\Psi$-MT structures suggest that PKS $C$-MTs may share a similar position within a module as the $\Psi$-MT within the mFAS.

\section{Experimental Procedures}

\section{Construct Design, Protein Expression, and Purification}

All PCR primers are listed in Supplementary Table 3. CurJ C-MT (CurJ residues 12691658) was amplified from cosmid pLM9 (GenBank accession code AY652953) ${ }^{8}$. JamJ $C$ MT (residues 1948-2337) and ACP (residues 3166-3302) were amplified from a mixture of jamacamide A cosmids ${ }^{9}$. $C$-MT and ACP constructs were inserted into pMCSG7 by ligation independent cloning (LIC) to create pMAS179 (CurJ $C$-MT), pMAS195 (JamJ $C$-MT), and pMAS197 (JamJ ACP). The QuikChange protocol (Stratagene) was used for site-directed mutagenesis. DNA sequencing at the UM DNA Sequencing Core verified all constructs and 
mutations. $C$-MT and ACP constructs were recombinantly expressed in Escherichia coli and purified using nickel affinity followed by gel filtration chromatography.

\section{Protein Crystallization and Structure Determination}

CurJ $C$-MT (native and SeMet) was crystallized by vapor diffusion from 2:2 $\mu \mathrm{L}$ ratio of protein stock $(10 \mathrm{mg} / \mathrm{mL}$ of CurJ $C$-MT in buffer C with $1 \mathrm{mM} \mathrm{SAM}$ or SAH) and well solution (1.30-1.38 M sodium citrate, 4-9 mM glutathione (GSH)/glutathione disulfide (GSSG), $5 \%(\mathrm{v} / \mathrm{v})$ acetone) at $20^{\circ} \mathrm{C}$. Thin rod-like crystals appeared in a few hours and grew overnight. Crystals were harvested directly from the drop and flash cooled in liquid nitrogen. Single-wavelength anomalous diffraction was used to determine the CurJ $C$-MT structure. A fully refined SeMet structure was used to solve the structure of native CurJ $C$-MT crystals.

\section{Enzyme Assays}

Reaction mixtures contained $25 \mu \mathrm{M}$ enzyme (CurJ C-MT, JamJ C-MT, or variants), $100 \mu \mathrm{M}$ JamJ ACP, $10 \mu \mathrm{M}$ Streptomcyes verticillus phosphopantethinyl transferase (SVP) ${ }^{49}, 1 \mathrm{mM}$ $\mathrm{MgCl}_{2}, 1 \mathrm{mM}$ SAM, and $500 \mu \mathrm{M}$ malonyl-CoA, acetoacetyl-CoA, or DL- $\beta$-hydroxybutyrylCoA in $50 \mathrm{mM}$ HEPES pH 7 (phosphopantetheine ejection) or 7.4 (intact protein), $150 \mathrm{mM}$ $\mathrm{NaCl}$ (total volume $50 \mu \mathrm{L}$ ). Reactions were incubated at $30^{\circ} \mathrm{C}$ for $6 \mathrm{hr}$ (phosphopantetheine ejection) or overnight (intact protein) and quenched with $1 \%$ (v/v) formic acid. JamJ ACP from the jamaicamide pathway of another Moorea producens $\operatorname{strain}^{9}$ (82\% identical to CurJ $\mathrm{ACP})$ was used as a surrogate due to the toxicity of CurJ ACP to $E$. coll ${ }^{35}$. Nearly identical levels of activity were observed for CurJ and JamJ $C$-MT (Figure 2f), indicating that the JamJ ACP is a suitable surrogate for CurJ ACP. NAC-linked substrates $(\mathbf{5}, \mathbf{7})$ were synthesized as previously described ${ }^{50}$. For NAC-linked substrate reactions $25 \mu \mathrm{M} \mathrm{C}$-MT was incubated overnight at $25^{\circ} \mathrm{C}$ with $1 \mathrm{mM} \mathrm{SAM}$ and $500 \mu \mathrm{M} 5$ or 7 in $50 \mathrm{mM}$ HEPES 7.4, $150 \mathrm{mM} \mathrm{NaCl}(50 \mu \mathrm{L})$. Reaction mixtures were quenched with $150 \mu \mathrm{L}$ methanol. Portions of the reaction mixes for ACP-linked substrates $(10 \mu \mathrm{L})$ and NAC-linked substrates $(20 \mu \mathrm{L})$ were used for LC-MS analysis (Agilent 6520 Accurate Mass Q-TOF in the positive mode equipped with an Agilent 1290 HPLC system). Separation of samples for intact protein analysis and phosphopantetheine ejection was performed using reverse phase chromatography (Aeris widepore $\mathrm{C} 4$ column $3.6 \mu \mathrm{m}, 50 \times 2.10 \mathrm{~mm}$ ) at a flow rate of 0.5 $\mathrm{mL} / \mathrm{min}$. Protein was eluted using a gradient of $5-100 \% \mathrm{~B}$ over $4 \min \left(\mathrm{A}-\mathrm{H}_{2} \mathrm{O}\right.$ with $0.2 \%$ $(\mathrm{v} / \mathrm{v})$ formic acid, B- acetonitrile with $0.2 \%$ (v/v) formic acid). Intact protein data were analyzed using the Agilent Mass Hunter Qualitative Analysis software with the maximum entropy deconvolution algorithm. Abundance of holo, acetoacetyl, and methylated acetoacetyl phosphopantetheine ejection masses were used to determine percent conversion of CurJ $C$-MT variants ${ }^{34}$. Data were normalized to exclude the spontaneous hydrolysis product (holo-ACP) of acetoacetyl or a-methyl-acetoacetyl-ACP. Three independent reactions were conducted and analyzed for each $C$-MT variant in the phosphopantetheine ejection assay. NAC-linked reaction mixes were separated using a Phenomenex Kinetix reverse-phase $\mathrm{C} 18$ column $(40 \mathrm{~mm} \times 2.1 \mathrm{~mm}, 2.6 \mu \mathrm{m})$ at a flow rate of $0.5 \mathrm{~mL} / \mathrm{min}$. Substrates were eluted using a gradient of $5-100 \%$ B over 4 min $\left(A-\mathrm{H}_{2} \mathrm{O}\right.$ with $0.2 \%$ (v/v) formic acid, B- acetonitrile with $0.2 \%$ (v/v) formic acid). 


\section{Supplementary Material}

Refer to Web version on PubMed Central for supplementary material.

\section{Acknowledgments}

This work was supported by NIH grants DK042303 to J.L.S., CA108874 to D.H.S., W.H.G. and J.L.S., and GM118101 to D.H.S. M.A.S. was supported by a predoctoral fellowship from the Cellular Biotechnology Training Program (T32GM008353) and Rackham Graduate School. GM/CA@APS is supported by the NIH National Institute of General Medical Sciences (AGM-12006) and National Cancer Institute (ACB-12002). The Advanced Photon Source is a US Department of Energy (DOE) Office of Science User Facility operated by Argonne National Laboratory under Contract No. DE-AC02-06CH11357.

\section{References}

1. Cox RJ. Polyketides, proteins and genes in fungi: programmed nano-machines begin to reveal their secrets. Org Biomol Chem. 2007; 5:2010-2026. [PubMed: 17581644]

2. Keatinge-Clay AT. The structures of type I polyketide synthases. Nat Prod Rep. 2012; 29:1050 1073. [PubMed: 22858605]

3. Smith S, Tsai SC. The type I fatty acid and polyketide synthases: a tale of two megasynthases. Nat Prod Rep. 2007; 24:1041-1072. [PubMed: 17898897]

4. Crawford JM, Townsend CA. New insights into the formation of fungal aromatic polyketides. Nat Rev Microbiol. 2010; 8:879-889. [PubMed: 21079635]

5. Gehring AM, DeMoll E, Fetherston JD, Mori I, Mayhew GF, Blattner FR, Walsh CT, Perry RD. Iron acquisition in plague: modular logic in enzymatic biogenesis of yersiniabactin by Yersinia pestis. Chem Biol. 1998; 5:573-586. [PubMed: 9818149]

6. Hendrickson L, Davis CR, Roach C, Nguyen DK, Aldrich T, McAda PC, Reeves CD. Lovastatin biosynthesis in Aspergillus terreus: characterization of blocked mutants, enzyme activities and a multifunctional polyketide synthase gene. Chem Biol. 1999; 6:429-439. [PubMed: 10381407]

7. Kennedy J, Auclair K, Kendrew SG, Park C, Vederas JC, Hutchinson CR. Modulation of polyketide synthase activity by accessory proteins during lovastatin biosynthesis. Science. 1999; 284:13681372. [PubMed: 10334994]

8. Chang Z, Sitachitta N, Rossi JV, Roberts MA, Flatt PM, Jia J, Sherman DH, Gerwick WH. Biosynthetic pathway and gene cluster analysis of curacin A, an antitubulin natural product from the tropical marine cyanobacterium Lyngbya majuscula. J Nat Prod. 2004; 67:1356-1367. [PubMed: 15332855]

9. Edwards DJ, Marquez BL, Nogle LM, McPhail K, Goeger DE, Roberts MA, Gerwick WH. Structure and biosynthesis of the jamaicamides, new mixed polyketide-peptide neurotoxins from the marine cyanobacterium Lyngbya majuscula. Chem Biol. 2004; 11:817-833. [PubMed: 15217615]

10. Magarvey NA, Beck ZQ, Golakoti T, Ding Y, Huber U, Hemscheidt TK, Abelson D, Moore RE, Sherman DH. Biosynthetic characterization and chemoenzymatic assembly of the cryptophycins. Potent anticancer agents from cyanobionts. ACS Chem Biol. 2006; 1:766-779. [PubMed: 17240975]

11. Ramaswamy AV, Sorrels CM, Gerwick WH. Cloning and biochemical characterization of the hectochlorin biosynthetic gene cluster from the marine cyanobacterium Lyngbya majuscula. J Nat Prod. 2007; 70:1977-1986. [PubMed: 18001088]

12. Sudek S, Lopanik NB, Waggoner LE, Hildebrand M, Anderson C, Liu H, Patel A, Sherman DH, Haygood MG. Identification of the putative bryostatin polyketide synthase gene cluster from "Candidatus Endobugula sertula", the uncultivated microbial symbiont of the marine bryozoan Bugula neritina. J Nat Prod. 2007; 70:67-74. [PubMed: 17253852]

13. Tillett D, Dittmann E, Erhard M, von Dohren H, Borner T, Neilan BA. Structural organization of microcystin biosynthesis in Microcystis aeruginosa PCC7806: an integrated peptide-polyketide synthetase system. Chem Biol. 2000; 7:753-764. [PubMed: 11033079]

14. Winter JM, Sato M, Sugimoto S, Chiou G, Garg NK, Tang Y, Watanabe K. Identification and characterization of the chaetoviridin and chaetomugilin gene cluster in Chaetomium globosum 
reveal dual functions of an iterative highly-reducing polyketide synthase. J Am Chem Soc. 2012; 134:17900-17903. [PubMed: 23072467]

15. Young J, Stevens DC, Carmichael R, Tan J, Rachid S, Boddy CN, Muller R, Taylor RE. Elucidation of gephyronic acid biosynthetic pathway revealed unexpected SAM-dependent methylations. J Nat Prod. 2013; 76:2269-2276. [PubMed: 24298873]

16. Tang L, Shah S, Chung L, Carney J, Katz L, Khosla C, Julien B. Cloning and heterologous expression of the epothilone gene cluster. Science. 2000; 287:640-642. [PubMed: 10649995]

17. Ansari MZ, Sharma J, Gokhale RS, Mohanty D. In silico analysis of methyltransferase domains involved in biosynthesis of secondary metabolites. BMC Bioinformatics. 2008; 9:454. [PubMed: 18950525]

18. Liscombe DK, Louie GV, Noel JP. Architectures, mechanisms and molecular evolution of natural product methyltransferases. Nat Prod Rep. 2012; 29:1238-1250. [PubMed: 22850796]

19. Kozbial PZ, Mushegian AR. Natural history of S-adenosylmethionine-binding proteins. BMC Struct Biol. 2005; 5:19. [PubMed: 16225687]

20. Maier T, Leibundgut M, Ban N. The crystal structure of a mammalian fatty acid synthase. Science. 2008; 321:1315-1322. [PubMed: 18772430]

21. Cacho RA, Thuss J, Xu W, Sanichar R, Gao Z, Nguyen A, Vederas JC, Tang Y. Understanding Programming of Fungal Iterative Polyketide Synthases: The Biochemical Basis for Regioselectivity by the Methyltransferase Domain in the Lovastatin Megasynthase. J Am Chem Soc. 2015; 137:15688-15691. [PubMed: 26630357]

22. Miller DA, Luo L, Hillson N, Keating TA, Walsh CT. Yersiniabactin synthetase: a four-protein assembly line producing the nonribosomal peptide/polyketide hybrid siderophore of Yersinia pestis. Chem Biol. 2002; 9:333-344. [PubMed: 11927258]

23. Poust S, Phelan RM, Deng K, Katz L, Petzold CJ, Keasling JD. Divergent mechanistic routes for the formation of gem-dimethyl groups in the biosynthesis of complex polyketides. Angew Chem Int Ed Engl. 2015; 54:2370-2373. [PubMed: 25564997]

24. Stevens DC, Wagner DT, Manion HR, Alexander BK, Keatinge-Clay AT. Methyltransferases excised from trans-AT polyketide synthases operate on $\mathrm{N}$-acetylcysteamine-bound substrates. J Antibiot (Tokyo). 2016

25. Wagner DT, Stevens DC, Mehaffey MR, Manion HR, Taylor RE, Brodbelt JS, Keatinge-Clay AT. a-Methylation Follows Condensation in the Gephyronic Acid Modular Polyketide Synthase. Chem Commun (Camb). 2016

26. Gerwick WH, Proteau PJ, Nagle DG, Hamel E, Blokin A, Slate DL. Structure of curacin A, a novel antimitotic, antiproliferative, and brine shrimp toxic natural product from the marine cyanobacterium Lyngbya majuscula. J Org Chem. 1994; 59:1243-1245.

27. Blokhin AV, Yoo H, Geralds RS, Nagle DG, Gerwick WH, Hamel E. Characterization of the Interaction of the Marine Cyanobacterial Natural Prouduct Curacin A with the Colchicine Site of Tubulin and Initial Structure-Activity Studies iwth Analogues. Mol Pharmacol. 1995

28. Akey DL, Razelun JR, Tehranisa J, Sherman DH, Gerwick WH, Smith JL. Crystal structures of dehydratase domains from the curacin polyketide biosynthetic pathway. Structure. 2010; 18:94105. [PubMed: 20152156]

29. Gehret JJ, Gu L, Gerwick WH, Wipf P, Sherman DH, Smith JL. Terminal alkene formation by the thioesterase of curacin A biosynthesis: structure of a decarboxylating thioesterase. J Biol Chem. 2011; 286:14445-14454. [PubMed: 21357626]

30. McCarthy JG, Eisman EB, Kulkarni S, Gerwick L, Gerwick WH, Wipf P, Sherman DH, Smith JL. Structural basis of functional group activation by sulfotransferases in complex metabolic pathways. ACS Chem Biol. 2012; 7:1994-2003. [PubMed: 22991895]

31. Gu L, Geders TW, Wang B, Gerwick WH, Hakansson K, Smith JL, Sherman DH. GNAT-like strategy for polyketide chain initiation. Science. 2007; 318:970-974. [PubMed: 17991863]

32. Gu L, Wang B, Kulkarni A, Geders TW, Grindberg RV, Gerwick L, Hakansson K, Wipf P, Smith JL, Gerwick WH, Sherman DH. Metamorphic enzyme assembly in polyketide diversification. Nature. 2009; 459:731-735. [PubMed: 19494914] 
33. Khare D, Hale WA, Tripathi A, Gu L, Sherman DH, Gerwick WH, Hakansson K, Smith JL. Structural Basis for Cyclopropanation by a Unique Enoyl-Acyl Carrier Protein Reductase. Structure. 2015; 23:2213-2223. [PubMed: 26526850]

34. Khare D, Wang B, Gu L, Razelun J, Sherman DH, Gerwick WH, Hakansson K, Smith JL. Conformational switch triggered by alpha-ketoglutarate in a halogenase of curacin A biosynthesis. Proc Natl Acad Sci U S A. 2010; 107:14099-14104. [PubMed: 20660778]

35. Whicher JR, Smaga SS, Hansen DA, Brown WC, Gerwick WH, Sherman DH, Smith JL. Cyanobacterial polyketide synthase docking domains: a tool for engineering natural product biosynthesis. Chem Biol. 2013; 20:1340-1351. [PubMed: 24183970]

36. Keatinge-Clay AT, Stroud RM. The structure of a ketoreductase determines the organization of the beta-carbon processing enzymes of modular polyketide synthases. Structure. 2006; 14:737-748. [PubMed: 16564177]

37. Herbst DA, Jakob RP, Zahringer F, Maier T. Mycocerosic acid synthase exemplifies the architecture of reducing polyketide synthases. Nature. 2016; 531:533-537. [PubMed: 26976449]

38. Piasecki SK, Zheng J, Axelrod AJ, Detelich ME, Keatinge-Clay AT. Structural and functional studies of a trans-acyltransferase polyketide assembly line enzyme that catalyzes stereoselective alpha- and beta-ketoreduction. Proteins. 2014; 82:2067-2077. [PubMed: 24634061]

39. Zheng J, Gay DC, Demeler B, White MA, Keatinge-Clay AT. Divergence of multimodular polyketide synthases revealed by a didomain structure. Nat Chem Biol. 2012; 8:615-621. [PubMed: 22634636]

40. Zeng J, Wagner DT, Zhang Z, Moretto L, Addison JD, Keatinge-Clay AT. Portability and structure of the four-helix bundle docking domains of trans-acyltransferase modular polyketide synthases. ACS Chem Biol. 2016

41. Zheng J, Taylor CA, Piasecki SK, Keatinge-Clay AT. Structural and functional analysis of A-type ketoreductases from the amphotericin modular polyketide synthase. Structure. 2010; 18:913-922. [PubMed: 20696392]

42. Zheng J, Piasecki SK, Keatinge-Clay AT. Structural studies of an A2-type modular polyketide synthase ketoreductase reveal features controlling alpha-substituent stereochemistry. ACS Chem Biol. 2013; 8:1964-1971. [PubMed: 23755878]

43. Zheng J, Fage CD, Demeler B, Hoffman DW, Keatinge-Clay AT. The missing linker: a dimerization motif located within polyketide synthase modules. ACS Chem Biol. 2013; 8:12631270. [PubMed: 23489133]

44. Keatinge-Clay AT. A tylosin ketoreductase reveals how chirality is determined in polyketides. Chem Biol. 2007; 14:898-908. [PubMed: 17719489]

45. Bonnett SA, Whicher JR, Papireddy K, Florova G, Smith JL, Reynolds KA. Structural and stereochemical analysis of a modular polyketide synthase ketoreductase domain required for the generation of a cis-alkene. Chem Biol. 2013; 20:772-783. [PubMed: 23790488]

46. Horowitz S, Dirk LM, Yesselman JD, Nimtz JS, Adhikari U, Mehl RA, Scheiner S, Houtz RL, AlHashimi HM, Trievel RC. Conservation and functional importance of carbon-oxygen hydrogen bonding in AdoMet-dependent methyltransferases. J Am Chem Soc. 2013; 135:15536-15548. [PubMed: 24093804]

47. Huang CC, Smith CV, Glickman MS, Jacobs WR Jr, Sacchettini JC. Crystal structures of mycolic acid cyclopropane synthases from Mycobacterium tuberculosis. J Biol Chem. 2002; 277:11559_ 11569. [PubMed: 11756461]

48. Meluzzi D, Zheng WH, Hensler M, Nizet V, Dorrestein PC. Top-down mass spectrometry on lowresolution instruments: characterization of phosphopantetheinylated carrier domains in polyketide and non-ribosomal biosynthetic pathways. Bioorg Med Chem Lett. 2008; 18:3107-3111. [PubMed: 18006314]

49. Sanchez C, Du L, Edwards DJ, Toney MD, Shen B. Cloning and characterization of a phosphopantetheinyl transferase from Streptomyces verticillus ATCC15003, the producer of the hybrid peptide-polyketide antitumor drug bleomycin. Chem Biol. 2001; 8:725-738. [PubMed: 11451672] 
50. Li Y, Dodge GJ, Fiers WD, Fecik RA, Smith JL, Aldrich CC. Functional Characterization of a Dehydratase Domain from the Pikromycin Polyketide Synthase. J Am Chem Soc. 2015; 137:7003-7006. [PubMed: 26027428] 
a.

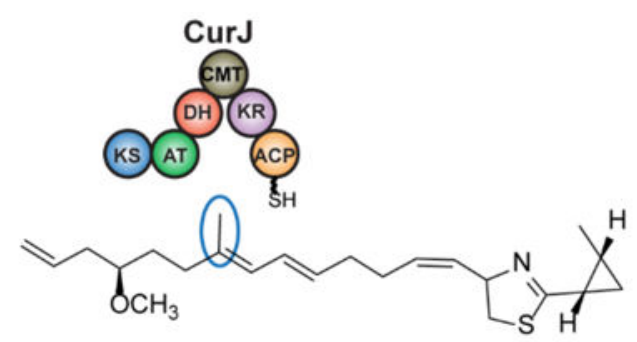

b.

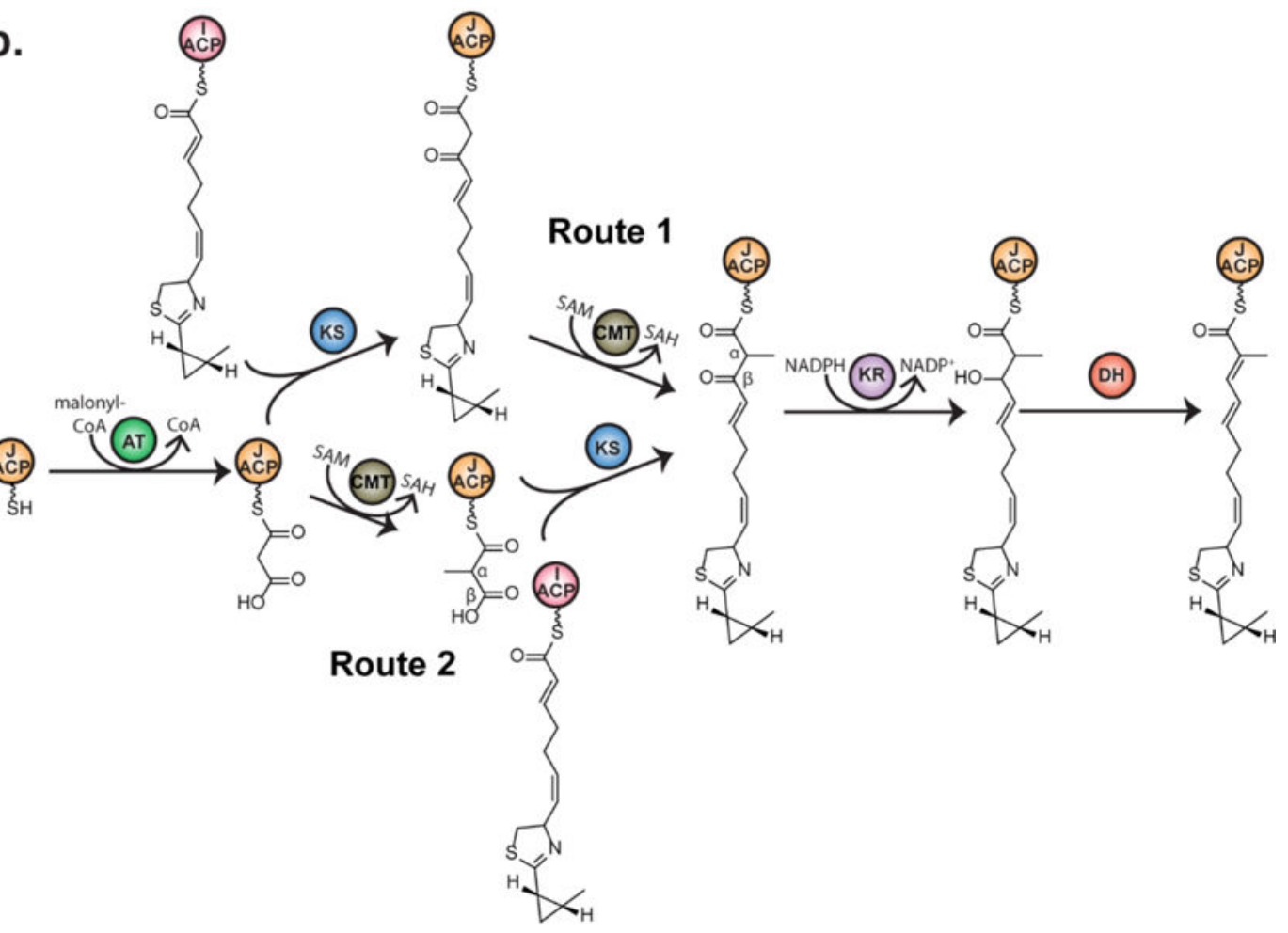

Figure 1.

Introduction of a-methyl by CurJ $C$-MT. a) CurJ composition. Curacin A contains a single methyl branch derived from the $C$-MT in module CurJ, which contains ketosynthase (KS), acyltransferase (AT), dehydratase (DH), $C$-methyltransferase (CMT), ketoreductase (KR), and acyl carrier protein (ACP) domains. b) Potential routes for $C$-methylation in PKSs. Route 1-Methylation occurs on the $\beta$ - ketoacyl intermediate after the KS condensation reaction. Route 2-Methylation occurs on malonyl-ACP prior to KS condensation. 

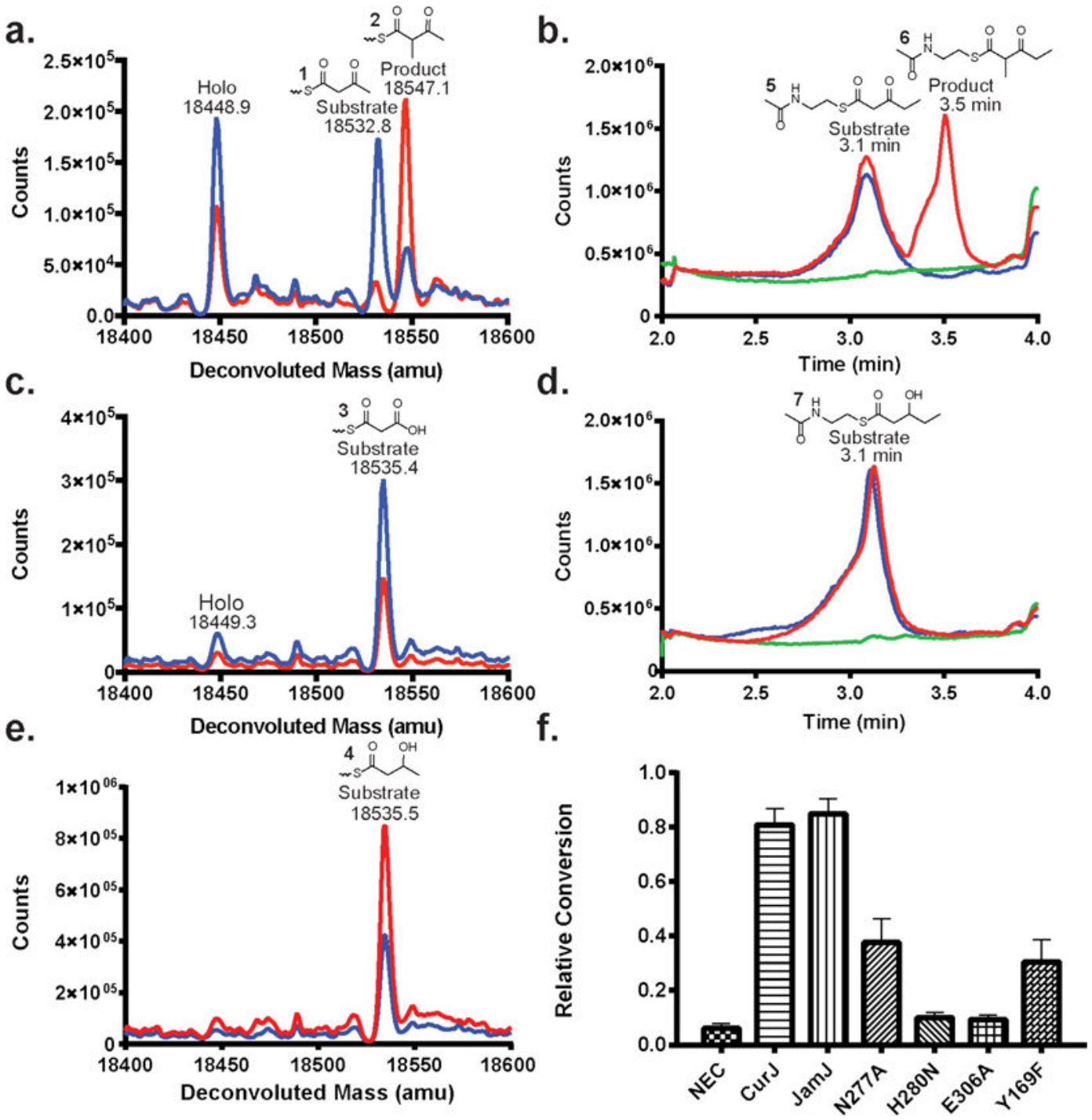

Figure 2.

LC-MS analysis of $C$-MT activity. Ion counts are shown in red for reactions via Route 1 in a) acetoacetyl-ACP (1) and b) $\beta$-ketopentanoyl-NAC (5), via Route 2 in c) malonyl-ACP (3), and via Route 3 in d) $\beta$-hydroxypentanoyl-NAC (7) and e) D,L- $\beta$-hydroxybutyryl-ACP (4). Blue traces are no-enzyme control reactions; green traces are no-substrate control reactions. See Supplementary Table 1 for expected masses and Supplementary Figure 3 for mass spectra of total ion chromatographs of acyl-NACs. f) Relative methylation activities of wild type CurJ $C$-MT, JamJ $C$-MT, and CurJ $C$-MT site-directed mutants with acetoacetyl-ACP (1) in an MS assay based on phosphopantetheine ejection (Supplementary Figure 5). NEC = no enzyme control. 
a.
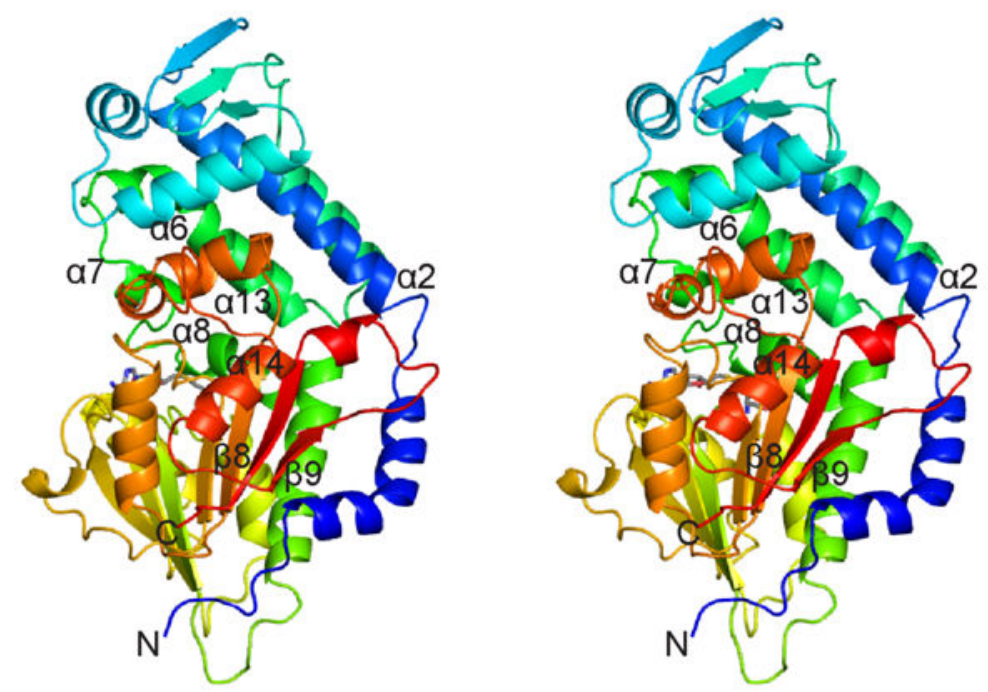

b.
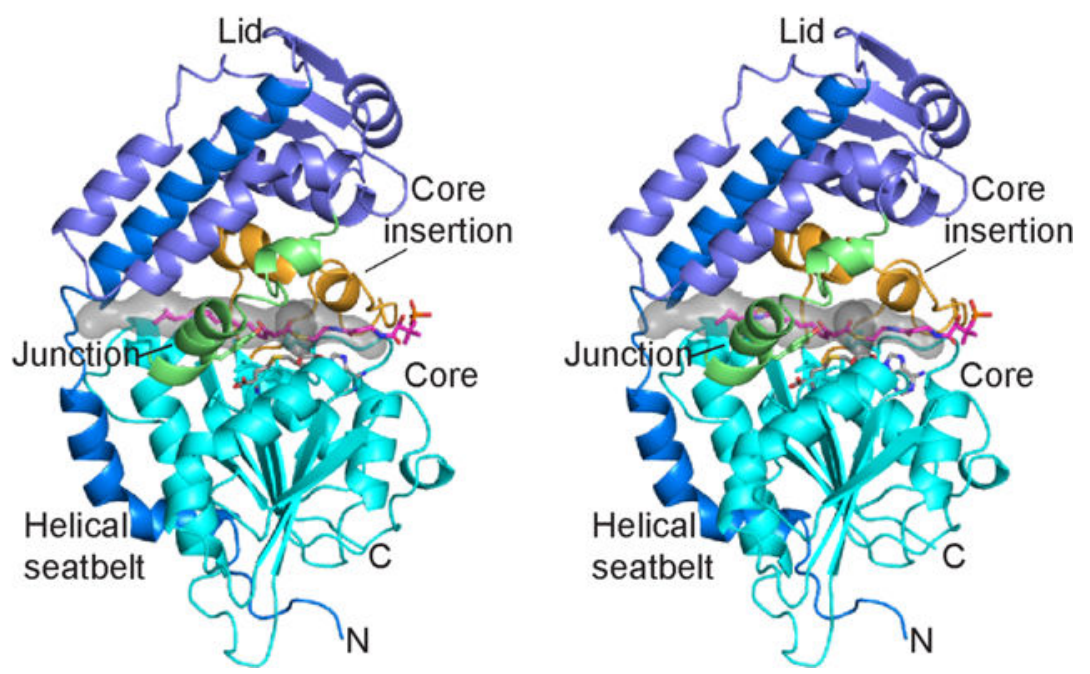

Figure 3.

CurJ $C$-MT structure. a) CurJ $C$-MT colored as a rainbow from blue (N-terminus) to red (Cterminus), shown in stereo. SAH is shown in sticks with atomic colors (C, gray; $\mathrm{O}$, red; $\mathrm{N}$, blue; S, yellow). b) CurJ $C$-MT colored by structure region. Helical seatbelt, blue; lid, dark blue; lid-to-core junction green; core, cyan; core insertion, orange; $\mathrm{SAH}$, sticks with gray $\mathrm{C}$. The transparent gray surface represents the substrate tunnel, which is lined with hydrophobic residues (Ile35, Phe157, Leu168, Val174, Ala307, Trp313, Val314, Phe318, Leu338). The CurJ $C$-MT substrate and phosphopantetheine (sticks with magenta $\mathrm{C}$ ) were modeled into the active site. The views in A and B are from opposite sides of the $C$-MT. 


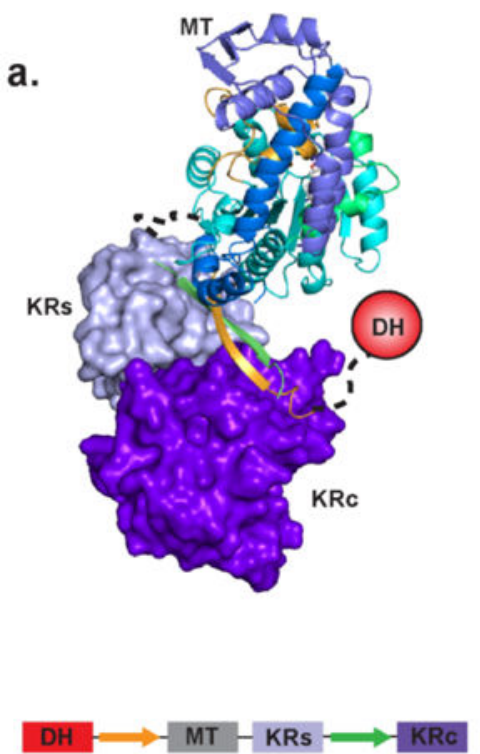

c.

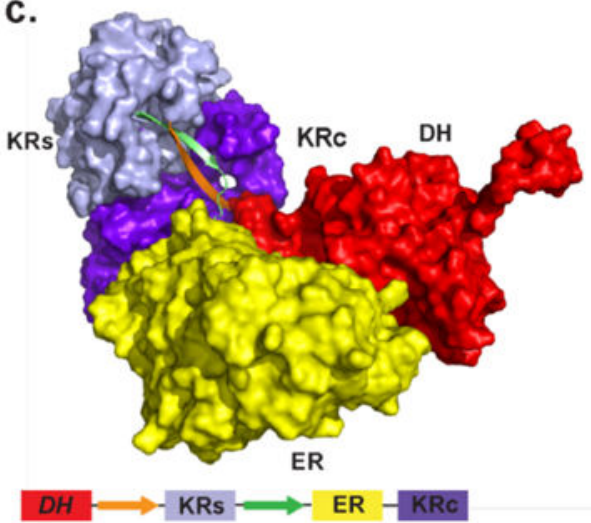

b.

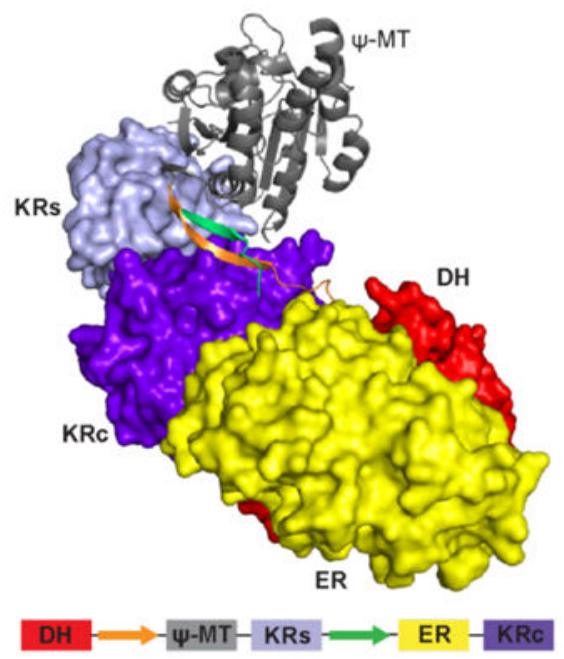

d.
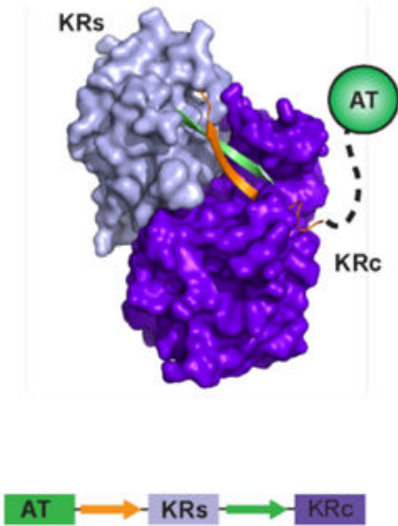

e.

CurJ $\mathrm{DH}_{1244}-9$ a.a. $-1254 \mathrm{~L}-\mathrm{Y}-\mathrm{E}-\mathrm{V}-\mathrm{E}-\mathrm{W}-\mathrm{R}-\mathrm{T}-8$ a.a. $-1270 \mathrm{C}-\mathrm{MT}-12$ a.a. - KRs

DEBS1 $\quad \mathrm{AT}_{1437}-15$ a.a. $-1453 \mathrm{R}-\mathrm{Y}-\mathrm{R}-\mathrm{I}-\mathrm{E}-\mathrm{W}-\mathrm{R}-\mathrm{P}-\mathrm{KR}_{\mathrm{s}}$

Plm1 $\quad \mathrm{DH}_{1803}-13$ a.a. $-1817 \mathrm{~W}-\mathrm{F}-\mathrm{S}-\mathrm{E}-\mathrm{T}-\mathrm{W}-\mathrm{R}-\mathrm{Q}-\mathrm{KR}$

SpnB $\quad \mathrm{DH}_{1209}-8$ a.a. $-1218 \mathrm{~L}-\mathrm{Y}-\mathrm{R}-\mathrm{L}-\mathrm{S}-\mathrm{W}-\mathrm{P}-\mathrm{T}-\mathrm{KR}$

MAS $\quad \mathrm{DH}_{1177}-14$ a.a. $-1192 \mathrm{~L}-\mathrm{L}-\mathrm{T}-\mathrm{I}-\mathrm{E}-\mathrm{W}-\mathrm{Q}-\mathrm{Q}-\mathrm{KR}_{\mathrm{s}}$

mFAS $\mathrm{DH}_{1104}-10$ a.a. $-1115 \mathrm{I}-\mathrm{L}-\mathrm{E}-\mathrm{K}-\mathrm{F}-\mathrm{C}-\mathrm{F}-\mathrm{T}-2$ a.a. $-1125 \mathrm{~T}-\mathrm{MT}-9$ a.a. $-\mathrm{KR}_{\mathrm{s}}$

Figure 4.

PKS and mFAS modifying regions. The $\beta$-ribbon that is both an essential part of the KR domain and also an inter-domain linker is orange ( $1^{\text {st }}$ strand) and green ( $2^{\text {nd }}$ strand). a) Model of CurJ $C$-MT and KR domains, arranged as in mFAS. The model is based on superpositions of the CurJ $C$-MT core with the mFAS $\Psi$-MT core and of a PKS KR with the mFAS KR ${ }^{20,36}$. b) mFAS with DH, $\Psi$-MT, ER and KR domains ${ }^{20}$. c) MAS-like PKS with DH, ER and KR domains ${ }^{37}$. d) DEBS1 module 1 modifying region with KR domain only ${ }^{36}$. $\mathrm{DH}$ (red), ER (yellow), $\mathrm{KR}_{\mathrm{C}}$ (purple) and $\mathrm{KR}_{\mathrm{S}}$ (light blue) domains are represented as surfaces, the CurJ $C$-MT as a ribbon colored as in Fig. 3b, the mFAS $\Psi$-MT as a gray ribbon, and other domains as circles. Below each structure is a schematic of domain and $\beta$ ribbon strand arrangement colored as in the structure image. e) Sequence alignment and 
domain context of $\beta$-ribbon strand 1 in CurJ, DEBS module $1^{36}$, phoslactomycin module $1^{45}$, spinosyn module $2^{39}$, MAS-like $\mathrm{PKS}^{37}$ and $\mathrm{mFAS}^{20}$. 


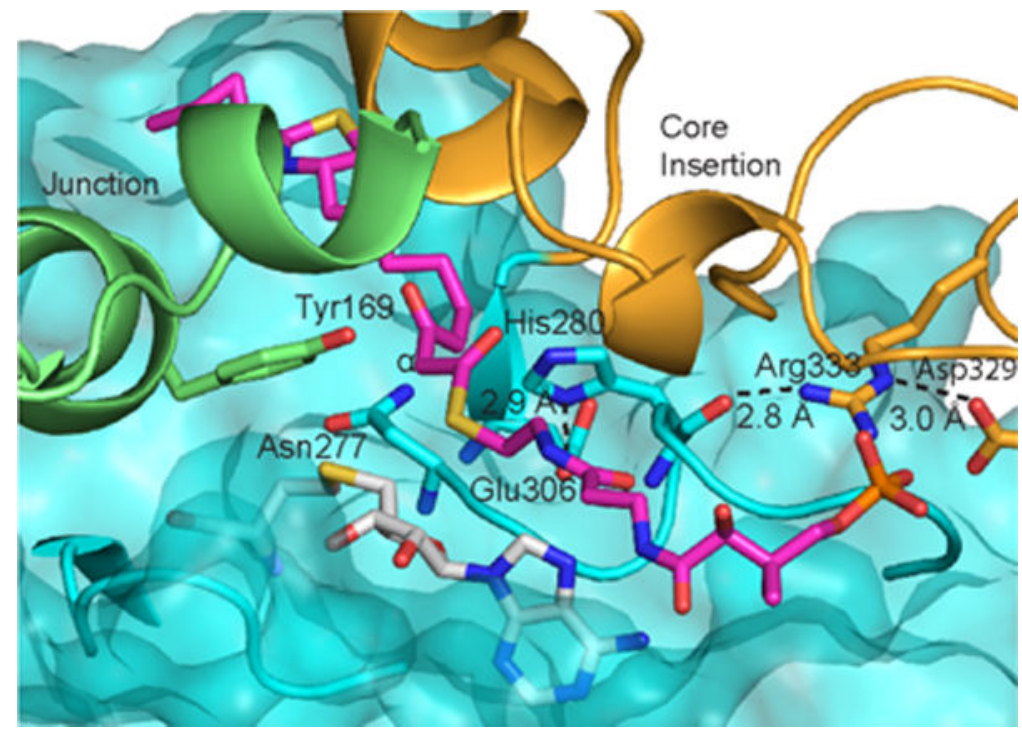

Figure 5.

CurJ $C$-MT active site. Key amino acids, modeled substrate (magenta $\mathrm{C}$ ), and SAH (white C) are shown in sticks. The MT core is represented as a transparent surface with ribbon. The junction and core insertion are shown as ribbons. $C$-MT structural regions are colored as in Fig. 3b. 

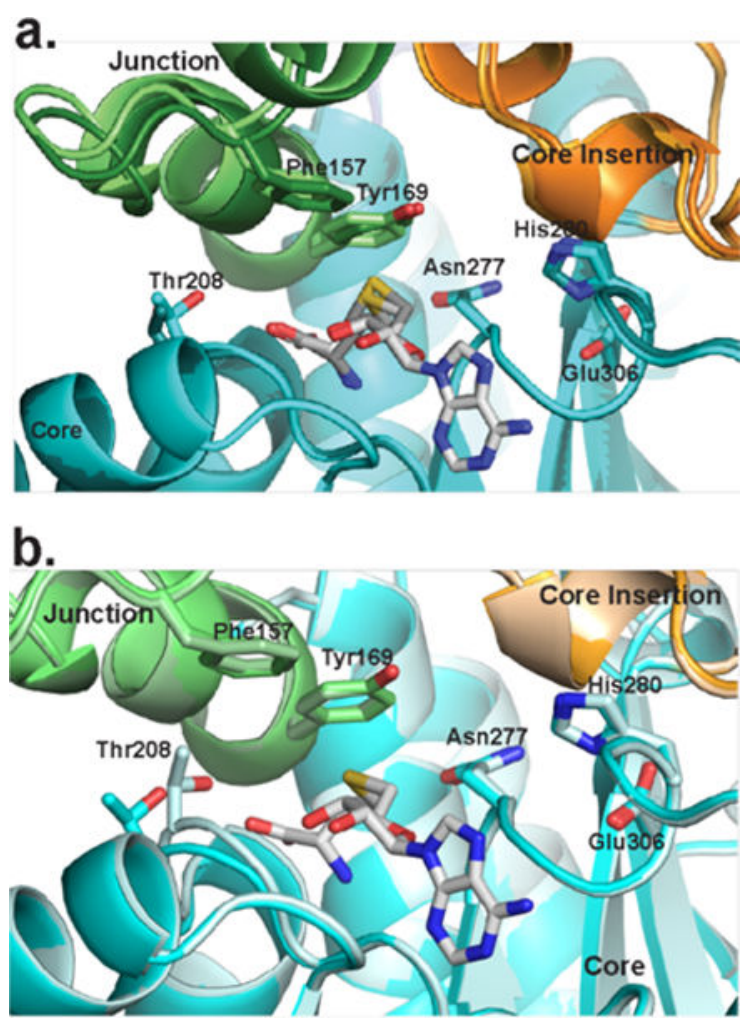

Figure 6.

Movement surrounding the CurJ $C$-MT active site. Structural regions are colored as in Fig. 3 b. a) Closely related SeMet and wild type CurJ $C$-MT crystal forms (light and dark colors) are different in the lid-to-core junction and SAH homocysteine position. b) The position of Thr208 in MT motif I differs in the two $C$-MT chains in the SeMet crystal form. 


\section{Table 1}

Crystallographic information

\begin{tabular}{|c|c|c|}
\hline Data Collection & SeMet & Wild type \\
\hline Space group & $P 2_{1}$ & $P 2_{1}$ \\
\hline \multicolumn{3}{|l|}{ Cell Dimensions } \\
\hline a,b,c $(\AA)$ & $48.4,130.4,62.8$ & $46.1,129.1,63.1$ \\
\hline$a, \beta, \gamma\left({ }^{\circ}\right)$ & $90,112.0,90$ & $90,111.5,90$ \\
\hline $\mathrm{X}$-ray Source & APS 23ID-D & APS 23ID-D \\
\hline Wavelength $(\AA)$ & 0.979 & 1.033 \\
\hline $\mathrm{d}_{\min }(\AA)$ & $2.09(2.16-2.09)^{a}$ & $2.10(2.18-2.10)$ \\
\hline R-merge & $0.1703(1.492)$ & $0.1508(1.048)$ \\
\hline Inner-shell R-merge & $0.053(9.3 \AA)^{b}$ & $0.049(6.2 \AA)^{b}$ \\
\hline $\operatorname{Avg} \mathrm{I} / \sigma(\mathrm{I})$ & $11.62(2.55)$ & $9.3(1.37)$ \\
\hline Completeness (\%) & $100(100)$ & $100(100)$ \\
\hline Multiplicity & $10.8(10.4)$ & $6.6(6.6)$ \\
\hline Total observations & $463,157(44,285)$ & $263,797(26,230)$ \\
\hline $\mathrm{CC}_{1 / 2}$ & $0.998(0.683)$ & $0.997(0.703)$ \\
\hline $\mathrm{CC}^{*}$ & $1(0.901)$ & $0.999(0.909)$ \\
\hline \multicolumn{3}{|l|}{ Refinement } \\
\hline Data range $(\AA)$ & $44.48-2.09$ & $43.43-2.10$ \\
\hline Reflections used in refinement & 42,794 & 39,893 \\
\hline $\mathrm{R}_{\mathrm{work}} / \mathrm{R}_{\text {free }}(\%)$ & $16.9 / 21.6$ & $18.5 / 25.2$ \\
\hline Number of non-hydrogen atoms & 6355 & 6220 \\
\hline macromolecules & 6049 & 6040 \\
\hline ligands & 40 & 19 \\
\hline water & 328 & 214 \\
\hline Amino acid residues & 765 & 765 \\
\hline \multicolumn{3}{|l|}{ Deviation from ideality } \\
\hline bond lengths $(\AA)$ & 0.003 & 0.007 \\
\hline bond angles $\left(^{\circ}\right)$ & 0.60 & 0.92 \\
\hline Average B-factor & 46.3 & 51.9 \\
\hline protein & 46.0 & 52.0 \\
\hline ligands & 86.3 & 70.3 \\
\hline solvent & 46.8 & 44.5 \\
\hline \multicolumn{3}{|l|}{ Ramachandran plot } \\
\hline favored $(\%)$ & 98 & 96 \\
\hline allowed (\%) & 2.5 & 3.4 \\
\hline outliers (\%) & 0 & 0.4 \\
\hline PDB & $5 \mathrm{THY}$ & $5 \mathrm{THZ}$ \\
\hline
\end{tabular}


${ }^{a}$ Values in parentheses pertain to the outermost shell of data.

$b_{\mathrm{d}_{\text {min }} \text { inner shell }}$ 